\title{
A Look at Fatalism
}

SCOTT STURGEON

Texas A \& M University

\section{Introduction}

Interestingly, fatalistic attitudes have played a significant role in the lives of men throughout the ages. From the Greek notion of destiny to Hitler's reliance on a personal astrologer, men have exhibited tendencies toward finding solace in at least some sort of fatalistic inclination. Yet few are those who adopt what could be termed a "proper" fatalistic outlook. This of course is the rather startling view that everything which in fact does happen could not have been otherwise. A less benign rendering of this view simply states that it is logically impossible for anything that does happen, not to happen. Obviously, adherers to this view think any sort of human deliberation pointless since they believe it not within their power to affect anything whatsoever. Everything that is, is simply because it logically must be; and as mentioned, few are willing to go so far as to accept this situation.

In fact, one interesting way to notice the force of this doctrine is to note the peculiar beliefs some philosophers have held in order to deny arguments in its support. Aristotle, for instance, claimed that future-tense contingent propositions are neither true nor false.' More recently, Prior has altered this approach slightly by stating that if $p$ is a contingent proposition, then it is false both that $p$ will be true and the negation of $p$ will be true. ${ }^{2}$ oddest of all is the very recent claim by Peter Geach that there are in fact no propositions about the future, but only propositions about the present tendencies of things." Further, Geach bases this position on the more substantive claim that the future is unreal, and thus there could be no propositions about it. Each of these positions marks a radical move in order to deny the doctrine of fatalism. Thus the purpose of this paper is to look at two fairly representative arguments for fatalism and refute them without appealing to a counter-intuitive metaphysical or logical construal. 


\section{I . The General Argument}

In order to adequately deal with the first argument, some general concepts will require preliminary explication." The concept of a "modal operator" greatly aids our inquiry and it is nothing more than this: the symbol "*" is introduced and thought of as governing a proposition ( $p)$. Further, the operator only correctly governs propositions that are considered to be "necessary truths"--for example:

*(all bachelors are single.)

or

$$
\star(4+4=8 .)
$$

This simply means that it is just not possible for these statements to be false. Now if the symbol "\#" is introduced and understood to designate possibility, a relationship between these two modal operators becomes apparent. This being:

$$
\text { If * }(\operatorname{not} p), \text { then } \operatorname{not}(\#(p)) \text {, }
$$

and conversely:

\section{If \# (not p), then not $(*(p))$.}

Also, the proposition inside the parentheses is said to "lie within the scope of the modal operator," and correctly determining a modal operator's scope is of vital importance. For example:

$$
\text { (A). * (If p, then q), }
$$

where the scope is the entire conditional, clearly functions in a different manner than

(B). If $P$, then * (q).

where the scope is just the consequent of the conditional. To see this, notice that conjoining $p$ with (A) does not allow one to validly infer * $(q)$, while conjoining $p$ with (B) does.

With all this in mind, then, consider the following fairly common argument for fatalism:

1) All projected future events either will obtain, or will fail to obtain.

2) If an event (E) will obtain, then it cannot be the case that (E) fails to obtain.

3) Event (E) will obtain.

4) Event (E) cannot fail to obtain.

5) Necessarily: Event (E) will obtain. 
Now going from the fact that a given event (E) will happen to the conclusion that (E) happens of necessity clearly signiffies this to be an argument for fatalism. But is it one we should accept? The first thing to notice is that the second premise is ambiguous. It can be read two ways, with one interpretation being trivial and the other being highly suspect, as follows:

2(a). Necessarily: If an event (E) will obtain, then it will obtain.

2(b). If an event (E) will obtain, then necessarily tt will obtain.

In the ambiguous version the word "cannot" functions as a modal operator signifying a non-possibility of one sort, and consequently, a necessity claim of a related sort. And it is in shifting the scope of this modal operator that gives rise to both interpretations of the premise. Obviously though, for the argument to be a convincing one the second premise has to be read as $2(a)$. For interpretation $2(b)$ is exactly the fatalist position, and reading the second premise as such reduces the entire "argument" to the trivial truth that "If fatalism is true, then fatalism is true."

However, if the second premise is read as 2 (a) then the argument is clearly invalid. For where an entire conditional proposition lies within the scope of a necessity claim, and the antecedent of the conditional is also given, one cannot validly infer the simple consequent of the conditional to lie within the scope of a necessity claim. That is, the argument form

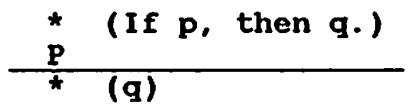

is invalid. But this is precisely what occurs when the second premise is read as $2(a)$. The confusion arises because in English, when the word "could" functions as a modal operator whose scope is an entire conditional, "could" may nevertheless be placed in a clause of the conditional. So our initial argument for fatalism is either trivial or invalid, and it 18 now time to move on the other argument for fatalism to be considered in this paper.

\section{Time Considerations and Richard Taylor}

our first argument dealt with fatalism concerning the future. In fact, fatalism concerning the past seems generally accepted by everyone." For if $t_{1}$ is earlier than $t_{2}$, there is a definite sense in which the obtainment of events at $t_{1}$ are in some way "necessary" at $t_{2}$. For example, it would seem highly presumptuous of me to claim I now have the ability to bring it about 
that some past event did not occur, i.e., to now keep Hitler from having been in power. It is for this reason that Aristotie writes:

- . no one deliberates about the past, but about what is future and capable of being otherwise, while what is past is not capable of not having taken place; hence Agathon is right in saying. "Eor this alone is lacking even to God/To make undone things that have once been done."

So fatalism with regard to the past seems obvious while fatalism with regard to the future arouses our interest and concern. But are there any arguments for fatalism with regard to the future that don't seem to commit invalid inferences? I believe Richard Taylor presents an argument of this sort that needs to be addressed." The presuppositions Taylor gives for his argument will be directly transcribed, while the rest of his argument will be paraphrased. The argument basically consists of two parts and goes as follows:

\section{Presuppositions}

A. We presuppose that any proposition whatever is either true or, if not true then false.

B. We presuppose that, if any state of affairs is sufficient for, though logically unrelated to, the occurence of some further condition at the same or any other time, then the former cannot occur without the latter occuring also.

c. We presuppose that, if the occurence of any condition is necessary for, but logically unrelated to, the occurence of some other condition at the same or any other time, then the latter cannot occur without the former occuring also.

D. We presuppose that, if one condition or set of conditions is sufficient for (ensures) another, then that other is necessary (essential) for it, and conversely, if one condition or set of conditions is necessary (essential) for another, then that other is sufficient for (ensures) it.

E. We presuppose that no agent can perform any given act if there is lacking, at the same or any other time, some condition necessary for the occurence of that act.

F. We presuppose that time is not by itself "efficacious": that is, that the mere passage of time does not augment or diminish the capacities of anything and, in particular, that it does not enhance or decrease an agent's powers or abilities. This means that if any substance or agent gains or loses powers or abilities over the course of time-such as, for instance, the power of a substance to corrode, or a man to do thirty push-ups, and so on--then such gain or 
loss is always the result of something other than the mere passage of time."

\section{ii. Argument}

Now let the statements "A naval battle will occur tomorrow" and "A naval battle will not occur tomorrow" be $Q$ and $Q^{\prime}$ respectively. Also, suppose $I$ am a naval commander about to issue an order to the fleet such that given all other prevalent conditions, the issuance of one sort of order ensures a naval battle tomorrow. while the issuance of another sort of order ensures that there will be no naval battle tomorrow. Let orders of the first sort be called 0 and orders of the second sort $O^{\prime}$.

Taylor wishes to block the non-fatalist claim

NE: It is in my power to do $O$, and it is also within my power to do $0^{\prime}$.

with the following argument:

1) If $Q$ is true, then it is not within my power to do of (for in case $Q$ is true, there is, or will be, lacking a condition essential for my doing $O^{\prime}$, the condition, namely, of there being no naval battle tomorrow).

2) But if $Q^{\prime}$ is true, then it is not within my power to do 0 (for a similar reason).

3) But either $Q$ is true, or $Q^{\prime}$ is true.

4) Either it is not within my power to do 0 , or it is not within my power to do $O^{\prime} .{ }^{10}$

In this argument step 4 obviously contradicts NE, thereby establishing fatalism concerning the future. The argument's form is valid so accepting its conclusion rests upon accepting Taylor's presuppositions and their implications. The line of attack I will adopt is similar to Lynne Spellman's position with regard to Taylor, and, like Spellman, I will begin with some preliminary remarks about causality. 11

Taylor's presupposition (E) claims that any substance or agent that gains or loses abilities does so as a result of something other than the mere passage of time. This "as a result of "may be interpreted as some sort of causality and the argument then might be attacked on the grounds that fatalism precludes causal efficacy. But does it?

If an event $y$ follows an event $x$, and as Taylor would claim both $x$ and $y$ are fated, can $x$ be said to cause $y$ ? Spellman thinks not for she asks, "If $x$ were to fail to occur (a situation which may be impossible, but not logically impossible and not meaningless). would y still occur? If it would, where is the causality? If it would not, where is the fatalism? For 
this reason I am inclined to agree with those who think that fatalism precludes causal efficacy."12 Here I think spellman misses the point completely. Fatalism is the doctrine that whatever events obtain, logically must obtain. So to grant that an event is fated, and then ask what the consequences would be if that event failed to obtain, is simply to ask an incolserent question. It is logically impossible and it is meaningless. Nonetheless, Taylor's position is stili problematic. It is my contention that Taylor borrows from two theories about the nature of time that are incompatible; that is, one cannot believe both theories to be true.

The first theory, known as the "A-theory," is more or less the common sense view of time. As G. N. Schlesinger notes," on this view the NOW is something that moves in a single direction relative to a series of temporal points that constitute all time. A given future instant along with everything that occurs then, steadily moves toward the NOW, momentarily is simultaneous with the NOW, and then forever steadily recedes from the Now. Temporal statements made in accordance with the A-theory are called "A-statements" and they contain the notions of pastness, presentness, and futurity. It is vitally important to notice that according to the A-theory some temporal statements change their truth value depending on when they are uttered. For example--"Jimmy Carter's election to the Fresidency is a past event." Here the A-theories simply claim that the truth value of this proposition is dependent upon when it is uttered. And for the A-theorist, this feature of A-statements is due to an ontological difference between the past and the future derived from the reiationship of the past and the future to the Now. In other words, A-statements change their truth value because the passage of time is an objective feature of reality being precisely described by the movement of the NOW.

The second theory of time, known as the "B-theory," is usually associated with the work of Bertrand Russell. On this view the universe does not contain the NOW as depicted by the A-theory, for the only temporal relations that exist are relations of earlier than or later than. There is no such thing as an event relative to the Now. Russell's claim, then, is that there are no A-statements. " The previous example would be translated as "Jimmy Carter's election to the Presidency is earlier than the utterance of this token," and this temporal relation, if ever true, is always true. So for the B-theorist no temporal statement changes its truth value because all temporal relations ( $x$ is earlier than, later than) are external. Consequently. there is no ontological difference between the past and the future; and the passage of time. or the movement of 
the NOW, is nothing more than a psychological aspect of the human organism. 23

It is easily seen that I have not tried to establish the superiority of the A-theory or the B-theory. The sole purpose in explicating these positions is to establish that one cannot hold both to be true. With this in mind and some careful scrutiny, Taylor's argument readily appears as implicitly unsound.

Notice in Taylor's presupposition (F) the statement that "the mere passage of time does not augment or diminish the capacities of anything." Erom this statement we can straightforwardly characterize Taylor as an A-theorist. For the B-theorist could make no sense out of talk about "the mere passage of time." In fact, the B-theory claims that all temporal points are equally real, and that time's passage is actually nonsense. obviously then, by denying efficacy of any sort to the "mere passage of time" Taylor's presupposition (E) commits him, ipso facto, to an A-theorist's perspective. And with the A-theory, remember, goes the notion of an ontological difference between the past and the future. This difference gives rise to the significant fact that for the A-theorist, the nature of the correlates (facts, states of affairs, etc.) to which true tensed propositions correspond varies in time--with the variance being a function of the time the correlate is actually under consideration. This construal, however, makes temporal relations between necessary and sufficient conditions highly relevant. For if a state of affairs taken. to be necessary for some other state of affairs is referred to in a tensed proposition, then the temporal relation indexed in that proposition is essential for determining whether the necessity claim is actually binding at the time of the proposition's utterance. And it is at this point that Taylor's problem moves to the forefront.

Instead of making temporal relations between necessary and sufficient conditions relevant, as a consistent A-theorist would, Taylor instead denies this in presupposition (E) where he states that "no agent can perform any given act if there is lacking, at the same or any other time. some condition necessary for the occurence of that act." What this means is that for any true tensed proposition referring to a necessary condition, the nature of such a proposition's correlate does not vary as a function of time--thus rendering the necessity claim equally applicable at all times. As Spellman correctly notes, 16 presupposition (E) entails the irrelevance of temporal relations between necessary and sufficient conditions.

But presupposition (E) is not the position of an Atheorist but rather that of a B-theorist. For an Atheorist would claim that any state of affairs' pastness or futurity is an ontological quality donned and doffed through the process of temporal becoming; and 
thus that temporal relations between such states of affairs and propositions referring to them are extremely important for evaluating the truth value of the propositions (or if you will, the applicability of a necessity claim relative to the utterance of such a claim). Not only would a consistent $A$-theorist shy away from Taylor's presupposition (E), he would reject it altogether. Obviously then Taylor wishes to claim on the one hand that temporal becoming is an objective feature of the world (presupp. (E)), and on the other hand that temporal relations between necessary and sufficient conditions are irrelevant (presupp. (E)). Thus we are forced to conclude that Taylor draws from both the $A$ and B-theories in constructing his argument, thereby rendering it implicitly unsound and unacceptable. For though presuppositions $(A)$ through (F) seem initially compelling, closer inspection reveals their dependence on Incompatible claims about the nature of time.

\section{Conclusion}

We began our discussion by noting the rather absolute character carried by the doctrine of fatalism. Some radical views were then mentioned displaying the extent to which some have gone in order to deny the doctrine. Two arguments for fatalism were then considered and rejected. The first argument was shown to be ambiguous with regard to the scope of its modal operator, with one interpretation rendering it trival and the other rendering it invalid. The second argument was accepted as valid, but its presuppositions were shown to rely on incompatible claims about the nature of time. In neither case was it necessary to make a radical move such as the denial of traditonal two-place logic, or the denial of a future altogether, in order to refute the fatalist position. Indeed, I believe no such argument exists that would necessitate such a move, but a defense of this claim is outside the scope of this paper.

\section{NOTES}

${ }^{1}$ De Interpretatione, chapter 11 .

${ }^{2}$ A. N. Prior, Past, Present, and Euture loxford, 1967), pp. 128-36.

'Peter Geach, Providence and Evil (Cambridge, 1979), pp. 52-53. 
"Eor a similar account, though used to attack physical determinism, see S. N. Thomas, "A Modal Muddle," Determinism, Eree Will, and Moral Responsibility (Prentice-Hali, 1970), pp. 141-48.

'For the sake of simplicity, fatalism with regard to the present will not be dealt with.

Eor a clear explication of this sort of "necessity," see A. J. Ereddoso, "Accidental Necessity and Power Over the Past," Pacific Philosophical Quarterly. 63 (1982), pp. 56-58.

'Nichomachean Ethics, VI, 2, 1139b.

"Richard Taylor, "Fatalism," The Philosophical Review, 71 (1962), pp. 56-66.

Ibid.. pp. 57-58.

${ }^{10}$ Ibid. . p. 61 .

"Lynne Speliman, "Forthcoming Sea Eights: What. Theory of Time?". The New Scholasticism, 55 (1981), pp. 52-54.

12 Ibid. . p. 54.

${ }^{3} \mathrm{G}$. N. Schlesinger, Aspects of Time (Hackett, 1980), p. 23.

'Bertrand Russell, Principles of Mathematics (Norton, 1937) Section 442 .

25instein also agrees with the concept of temporal becoming as psychological, but for different reasons. See his Relativity (Crown, 1961), pp. 139-40.

"Spellman, "Eorthcoming Sea Fights: What Theory of Time?", p. 61 . 